\title{
Breakdown of Photon Blockade: A Dissipative Quantum Phase Transition in Zero Dimensions
}

\author{
H. J. Carmichael \\ The Dodd-Walls Centre for Photonic and Quantum Technologies, Department of Physics, \\ University of Auckland, Private Bag 92019 Auckland, New Zealand
}

(Received 22 January 2015; published 8 September 2015)

\begin{abstract}
The Jaynes-Cummings model with coherent drive is considered as an example of a nonlinear oscillator exhibiting photon blockade, where blockade by one, two, three, etc., photons occurs at a sequence of multiphoton absorption resonances. It is shown that with increasing drive strength, the blockade breaks down by way of a dissipative quantum phase transition. The transition is first order, except at a critical point in the space of drive amplitude and detuning, where a continuous transition is observed. Numerical solutions to the quantum master equation in the steady state are presented and compared with mean-field treatments based on Jaynes and Cummings' semiclassical equations (strong coupling with conserved pseudospin) and the Maxwell-Bloch equations (spontaneous emission included). The concept of a "thermodynamic limit" in the absence of conserved particle number is explored. Contrasting the identity of large photon number with weak coupling (large volume) in other dissipative quantum phase transitions for photons (e.g., in the phase-transition analogy of laser threshold), the limit of large photon numbers is a strong-coupling limit.
\end{abstract}

DOI: 10.1103/PhysRevX.5.031028

Subject Areas: Photonics, Quantum Physics, Statistical Physics

\section{INTRODUCTION}

The unique $\sqrt{n}$ splitting of energy levels in the JaynesCummings model [1] (see also Ref. [2]) is a benchmark of quantum optics and the target of beautiful experiments seeking spectroscopic evidence for the quantization of the electromagnetic field [3-8]. The same $\sqrt{n}$ splitting is seen exchanging phonons with the center-of-mass motion of a trapped ion through Raman coupling of its electronic ground states [9]. There is more than a little irony to this legacy of a paper, which, published under the title "Comparison of Quantum and Semiclassical Radiation Theories ...," lays more emphasis on the successes of semiclassical theory than the need for something fully quantum. Indeed, Jaynes and Cumming's semiclassical ideas were developed over the period of a decade as neoclassical radiation theory [10] and viewed with enough seriousness to be put to the experimental test $[11,12]$.

I do not aim to revisit the quantum versus semiclassical issue in this paper. Nonetheless, neoclassical radiation theory is inevitably a subtext to a report of a quantum phase transition of the Jaynes-Cummings model.

The notion of photon blockade was introduced by Imamoğlu et al. [13] as an analogue of Coulomb blockade

Published by the American Physical Society under the terms of the Creative Commons Attribution 3.0 License. Further distribution of this work must maintain attribution to the author(s) and the published article's title, journal citation, and DOI. for quantum-well electrons. These authors treat an optical cavity with Kerr nonlinearity and say, "To explain the strong antibunching of transmitted photons, we introduce the concept of photon blockade in close analogy with the phenomenon of Coulomb blockade for quantum-well electrons." The essence of the blockade effect is that harmonic energy levels are sufficiently shifted for a cavity excited out of the ground state to behave as a two-state system. Its realization as a result of the $\sqrt{n}$ splitting of the Jaynes-Cummings model was anticipated by Tian and Carmichael [14], who note in the abstract of their 1992 paper, "Under conditions of strong dipole coupling an optical cavity containing one atom behaves as a two-state system when excited near one of the 'vacuum' Rabi resonances." With just one two-level system coupled to one radiation mode-i.e., for the Jaynes-Cummings model-the vacuum Rabi resonance is highly anharmonic: For dipole-coupling strength $g$, a coherent drive tuned to one-photon resonance out of the ground state is detuned by $\pm(\sqrt{2}-2) g$ from the second excited state; with this detuning much larger than the cavity width, absorption of one photon "blocks" absorption of a second.

Experiments in cavity and circuit QED have observed the blockade effect by measuring photon antibunching $[15,16]$ and Mollow triplet spectra [16] in transmission and "supersplitting" of the vacuum Rabi resonance as a function of increasing drive strength [8]. Realization of Gardiner's squeezing-inhibited decay of atomic coherence relies on photon blockade [17-19], and photon blockade in 
the Jaynes-Cummings model is key to proposals for phase transitions of light in photonic cavity arrays [20-22] in analogy to the superfluid-to-Mott-insulator quantum phase transition of the Bose-Hubbard model $[23,24]$.

With regard to the latter proposals, the analogy maps Mott phases of exactly one-, two-, three-, etc., particle occupancy per site to excited states of the JaynesCummings Hamiltonian occupied by one, two, three, etc., quanta, with the one-quantum state corresponding to the vacuum Rabi resonance where standard photon blockade is observed. Higher excited states are accessed as two-, three-, four-, etc., photon resonances [5,8,25], and, in a generalization of one-photon blockade, each such multiphoton resonance exhibits blockade, inhibiting the absorption of additional photons [26].

Photon blockade is not, however, an absolute barrier to the absorption of additional photons. It arises as an excitation bottleneck caused by detuning when one tries to drive monochromatic photons up an energy ladder of unequal steps. In principle, it can be broken through, since increasing drive strength both shifts and broadens energy levels, increasing the probability for multiphoton absorption up many steps of the ladder. I show in this paper that the breaking through - the breakdown of photon blockade - is organized around a dissipative quantum phase transition, an extension of what Alsing and Carmichael call spontaneous dressed-state polarization [27].

In their 1991 paper, Alsing and Carmichael report a symmetry-breaking transition of second order at a critical value of drive strength for zero drive detuning. The quasienergy spectrum of the driven Jaynes-Cummings model shows a singularity at the critical point [28], where the $\sqrt{n}$ splitting collapses to 0 ; a discrete spectrum below the critical point gives way to a continuous one above. In this paper, I show, more generally, that a first-order transition occurs at nonzero drive detuning, evidenced by the coexistence of the vacuum state with a state where the cavity is filled with light $[29,30]$ — photon numbers ranging in the hundreds for a dipole couple strength of $g / \kappa=50$, where $2 \kappa$ is the cavity width. Fluctuations cause the cavity transmission to "blink" on and off, with blink times on the order of a thousand cavity lifetimes (critical slowing down). By solving a quantum master equation numerically, I map out the dependence of the mean steady-state photon number on drive amplitude and detuning, and also the domain of coexistent states, as defined by a bimodal steadystate $Q$ function.

I recover an outline of the physics behind the numerical results from a mean-field treatment based on Jaynes and Cumming's semiclassical (neoclassical) radiation theory $[1,10]$, which serves as background to a discussion of fluctuations. On resonance, the mean-field nonlinearity diverges as the photon number goes to 0 , contrasting the more usual situation-e.g., a saturable nonlinearitywhere low photon numbers recover a linear limit.
Energy quantization removes the divergence. I show, thus, that photon blockade breaks down, not through a meanfield nonlinearity, but in a cascade of multiphoton transitions. Quantum fluctuations are central. I illustrate their role by comparing mean-field theory with master equation and quantum trajectory results.

In opposition, for example, to laser threshold [31-33] where weaker coupling means a bigger photon numberi.e., the "thermodynamic limit" is a weak-coupling limit [34] — photon numbers beyond the critical point are larger at stronger coupling and the "thermodynamic limit" is a strong-coupling limit. Finally, I show how the weakcoupling limit is restored by spontaneous emission, thus converting the breakdown of photon blockade into optical bistability for a saturable transition [35-38]. Although a source of large fluctuations when its rate is small (see, e.g., Sec. 5 of Ref. [27]), spontaneous emission of higher and higher rate restores a limit where fluctuations may be neglected. Through the agency of disentangling quantum jumps, distinguishable dressed-state ladders are replaced by separated entities, a two-state system and a radiation mode; environment-induced measurement transforms one phase transition into another.

Background material is reviewed in Sec. II. In Sec. III, I then survey the breakdown of photon blockade through numerical solutions to a quantum master equation in steady state: I map out the mean photon number and domain of coexistent states (bimodal $Q$ functions) in the plane of drive amplitude and detuning; the broad features of a dissipative quantum phase transition-critical point, bimodality, critical slowing down-are demonstrated and discussed. In order to target the breakdown of photon blockade in a clear and unambiguous way, spontaneous emission is omitted in Secs. II and III. The full quantum results of Sec. III are compared with mean-field theory in Sec. IV, where I also contrast strong-coupling and weak-coupling "thermodynamic limits"; spontaneous emission is introduced as the agent that connects them. I return to the numerical solution of the quantum master equation in steady state in Sec. V, now with spontaneous emission included. I conclude with a summary in Sec. VI, where I also discuss the potential extension from zero dimensions to a driven lattice.

\section{BACKGROUND}

Experimental observation of the superfluid-to-Mottinsulator transition in a gas of ultracold atoms [24] stimulated wide interest in quantum phase transitions beyond their traditional home in materials science. Such transitions are now a target of quantum simulation, in lattices of ultracold atoms and trapped ions, and photonic cavity and superconducting circuit arrays [39-42]. Attention has focused for the most part on thermal equilibrium transitions, where particle number is conserved. Photon number is not conserved, so while photonic models may imitate equilibrium predictions-e.g., those of 
the Bose-Hubbard model [20-22] — they lead more naturally in the direction of nonequilibrium (dissipative) physics. Notable experiments are the realization of the Dicke quantum phase transition [43-45] through photon scattering from a superfluid gas in an optical cavity [46] and the observation of Bose-Einstein condensation of photons in an optical microcavity [47]. Both address phase transitions first met in thermal equilibrium but nevertheless employ laser driving to overcome photon loss. The Dicke phase transition is realized in an explicitly dissipative version, with his "caricature" - to quote Refs. [44] and [48]—of the minimal coupling Hamiltonian engineered, in the interaction picture, from a coherently driven Raman configuration [49].

There is a superficial similarity between the driven Jaynes-Cummings Hamiltonian and the Hamiltonian of the Bose-Hubbard model in its mean-field approximation [50] (see Sec. II D). Beyond this, thermal equilibrium is set aside in this paper. The breakdown of photon blockade is a quantum phase transition in zero dimensions (one optical cavity rather than a cavity array) far away from thermal equilibrium. Phase transitions of this type have a long history in quantum optics, most often encountered as a rather elementary bifurcation at the mean-field (semiclassical) level. The most familiar example is the laser, whose threshold behavior was treated as a second-order phase transition far from thermal equilibrium in the early 1970s [31-33]. Lasers may also exhibit transitions of first order $[51,52]$, as in the much-studied example of optical bistability for a saturable transition [36-38]. Cooperative resonance fluorescence [53-55] is a driven version of the Dicke system and exhibits a second-order transition. The nonlinear physics upon which these and other examples rely is captured by mean-field equations; quantum mechanics adds only a small level of noise, e.g., the phase noise (Schawlow-Townes linewidth) of laser light. The breakdown of photon blockade differs in the interplay of mean-field nonlinearity and quantum noise, since the $\sqrt{n}$ splitting of Jaynes-Cummings energy levels corresponds to a mean-field nonlinearity that diverges at zero photon number. The consequences of the difference are presented in Secs. III and IV. I first review background topics.

\section{A. Photon blockade}

Throughout, I treat the Jaynes-Cummings model with the field mode and the two-state system on resonance, with frequency $\omega_{0}$. The well-known Hamiltonian is

$$
H_{\mathrm{JC}}=\hbar \omega_{0}\left(a^{\dagger} a+\sigma_{+} \sigma_{-}\right)+\hbar g\left(a \sigma_{+}+a^{\dagger} \sigma_{-}\right),
$$

where $a^{\dagger}$ and $a$ are creation and annihilation operators for the field, $\sigma_{+}$and $\sigma_{-}$are raising and lowering operators for the two-state system, and $g$ is the dipole-coupling strength. Diagonalization of the Hamiltonian yields the ground state $\left|E_{0}\right\rangle=|0,-\rangle$, with energy $E_{0}=0$, and excited-state doublets

$$
\begin{aligned}
& \left|E_{n, U}\right\rangle=\frac{1}{\sqrt{2}}(|n,-\rangle+|n-1,+\rangle), \\
& \left|E_{n, L}\right\rangle=\frac{1}{\sqrt{2}}(|n,-\rangle-|n-1,+\rangle),
\end{aligned}
$$

$n=1,2, \ldots$, with energies

$$
\begin{aligned}
& E_{n, U}=n \hbar \omega_{0}+\sqrt{n} \hbar g, \\
& E_{n, L}=n \hbar \omega_{0}-\sqrt{n} \hbar g .
\end{aligned}
$$

Photon blockade enters the picture when an external drive, frequency $\omega_{D}$, is tuned to a transition from the ground to the first excited state, $\hbar \omega_{D}=\hbar \omega_{0} \pm \hbar g$. From the $\sqrt{n}$ splitting, the next step up the ladder is detuned by $E_{2,(U, L)}-E_{1,(U, L)}-\hbar \omega_{D}=\mp(2-\sqrt{2}) \hbar g$. Thus, for sufficiently large dipole coupling compared to level widths, the Jaynes-Cummings system behaves as a two-state system when driven out of the ground state [14]. In a straightforward extension, a multiphoton blockade occurs if the drive is tuned to a multiphoton resonance out of the ground state, $n \hbar \omega_{D}=n \hbar \omega_{0} \pm \sqrt{n} \hbar g$ [26]. In this case, the detuning from the next step up the ladder is $E_{n+1,(U, L)}-E_{n,(U, L)}-\hbar \omega_{D}=\mp[(n+1) / \sqrt{n}-\sqrt{n+1}] \hbar g$.

\section{B. Jaynes-Cummings Hamiltonian with coherent drive}

The Jaynes-Cummings Hamiltonian with drive is time dependent-it is periodic with frequency $\omega_{D}$. By working in an interaction picture, the time dependence is removed. I consider driving of the cavity mode (a transformation treats driving of the two-state system [27]) where the interaction-picture Hamiltonian is

$$
\begin{aligned}
H_{\mathrm{JCD}}^{\mathrm{int}}= & -\hbar \Delta \omega\left(a^{\dagger} a+\sigma_{+} \sigma_{-}\right)+\hbar g\left(a \sigma_{+}+a^{\dagger} \sigma_{-}\right) \\
& +\hbar \mathcal{E}\left(a^{\dagger}+a\right),
\end{aligned}
$$

with drive amplitude $\mathcal{E}$ and drive detuning

$$
\Delta \omega=\omega_{D}-\omega_{0} .
$$

Eigenvalues of $H_{\mathrm{JCD}}^{\mathrm{int}}$ have the status of quasienergies, and their associated eigenkets are periodic solutions to the Schrödinger equation [56]. For a drive on resonance, $H_{\mathrm{JCD}}^{\text {int }}$ is diagonalized by a Bogoliubov transformation [28]. The quasienergy spectrum comprises an $e_{0}=0$ and quasienergy doublets

$$
e_{n,+}=+\sqrt{n} \hbar g\left[1-(2 \mathcal{E} / g)^{2}\right]^{3 / 4},
$$




$$
e_{n,-}=-\sqrt{n} \hbar g\left[1-(2 \mathcal{E} / g)^{2}\right]^{3 / 4},
$$

$n=1,2, \ldots$ Clearly, $\mathcal{E} / g \rightarrow 0$ recovers the usual JaynesCummings splitting. More generally, the quasienergy splittings shift inward and all collapse to 0 at $2 \mathcal{E}=g$, beyond which the quasienergy spectrum is continuous. The singularity locates a critical point-at $\Delta \omega=0$ and $2 \mathcal{E}=g-$ in the plane of drive amplitude and detuning. The critical point is the organizing center of a dissipative quantum phase transition (Secs. III and IV).

\section{Jaynes and Cummings semiclassical (neoclassical) equations}

The equations of Jaynes and Cummings' semiclassical radiation theory are reached by taking expectation values in Heisenberg equations of motion and factorizing the expectations of operator products. For one cavity mode, with loss at rate $\kappa$ included, they read:

$$
\begin{gathered}
\frac{d \alpha}{d t}=-(\kappa-i \Delta \omega) \alpha-i g \beta-i \mathcal{E}, \\
\frac{d \beta}{d t}=i \Delta \omega \beta+i g \alpha \zeta, \\
\frac{d \zeta}{d t}=2 i g\left(\alpha^{*} \beta-\alpha \beta^{*}\right),
\end{gathered}
$$

where $\alpha=\langle a\rangle, \beta=\left\langle\sigma_{-}\right\rangle$, and $\zeta=\left\langle\sigma_{z}\right\rangle=2\left\langle\sigma_{+} \sigma_{-}\right\rangle-1$. After Ref. [10], I call these neoclassical equations. Although it is today more common to speak of Maxwell-Bloch equations, I reserve that name for situations where the two-level system is also damped (Sec. IVA). Equations (11) and (12) conserve the length of the pseudospin $4|\beta|^{2}+\zeta^{2}=1$. The neoclassical equations are thus four rather than five equations, and with no driving - $\mathcal{E}=\Delta \omega=0$ - and the field adiabatically eliminated-solving Eq. (10) in steady state-they are reduced to an autonomous equation of motion for the inversion:

$$
\frac{d \zeta}{d t}=-\frac{g^{2}}{\kappa}\left(1-\zeta^{2}\right)
$$

In this fashion, the nonexponential decay of neoclassical radiation theory [see Eq. (3.24c) in Ref. [57]] is recovered.

Section IV compares a mean-field theory based on the neoclassical equations with the quantum treatment from Sec. III. The mean-field theory is built around steady-state solutions to Eqs. (10)-(12). Alsing and Carmichael [27] discuss these for resonant driving. In that case, with $\Delta \omega=0$, Eq. (11) requires either $\alpha=0$ or $\zeta=0$. Setting $\alpha=0$, Eq. (10) and the conserved value of pseudospin give

$$
\beta=-\mathcal{E} / g, \quad \zeta=\mp \sqrt{1-(2 \mathcal{E} / g)^{2}} .
$$

The solution holds so long as $\zeta$ remains real; thus, with increasing drive amplitude, we meet once again with the critical point at $\Delta \omega=0$ and $2 \mathcal{E}=g$. At higher drive amplitudes,

$$
\beta= \pm \frac{\alpha}{2|\alpha|}, \quad \zeta=0
$$

with $\alpha$ a solution to

$$
\alpha=-i \mathcal{E}\left(\kappa \pm i \frac{g}{2|\alpha|}\right)^{-1} .
$$

Passing through the critical point, the semiclassical state undergoes a parity-breaking transition: Above the critical point, the pseudospin sits at the equator of the Bloch sphere, where it adopts a phase either aligned or antialigned with the phase of the cavity field and no longer slaved to the phase of the drive. Alsing and Carmichael refer to spontaneous dressed-state polarization. When mapped onto the Jaynes-Cummings energy ladder, the two phases correspond to ascending the ladder either by its upper rungs $\left|E_{n-1, U}\right\rangle \rightarrow\left|E_{n, U}\right\rangle \rightarrow\left|E_{n+1, U}\right\rangle \cdots$ or lower rungs $\left|E_{n-1, L}\right\rangle \rightarrow$ $\left|E_{n, L}\right\rangle \rightarrow\left|E_{n+1, L}\right\rangle \cdots$

When the drive detuning is not $0, \Delta \omega \neq 0$, steady states of the neoclassical equations are given by

$$
\beta= \pm \operatorname{sgn}(\Delta \omega) \frac{g \alpha}{\sqrt{\Delta \omega^{2}+4 g^{2}|\alpha|^{2}}}
$$

and

$$
\zeta=\mp \sqrt{1-4|\beta|^{2}},
$$

where the field amplitude satisfies

$$
\alpha=-i \mathcal{E}\left[\kappa-i\left(\Delta \omega \mp \operatorname{sgn}(\Delta \omega) \frac{g^{2}}{\sqrt{\Delta \omega^{2}+4 g^{2}|\alpha|^{2}}}\right)\right]^{-1} .
$$

Clearly, Eq. (19) represents the response of a Lorentzian cavity resonance in the presence of a rather unusual-it diverges as $|\alpha|^{2} \rightarrow 0$ at $\Delta \omega=0$-nonlinear dispersion.

\section{Mean-field Hamiltonian of the photonic Bose-Hubbard model}

Before moving to results, it is important to draw a line between the breakdown of photon blockade - a dissipative quantum phase transition in zero dimensions-and the photon analogue of the Mott-insulator-to-superfluid quantum phase transition, which relates to a lattice of photonic cavities and is fundamentally an equilibrium transition [20-22]. Superficially, we appear to treat one lattice site and thus embark on a mean-field treatment of the 
Jaynes-Cummings analogue of the Bose-Hubbard system; this, in the mean-field decoupling approximation, has a single-site Hamiltonian (in units of $\hbar$ and suppressing the site index) [20,50]

$$
\begin{aligned}
H= & \left(\omega_{0}-\mu\right)\left(a^{\dagger} a+\sigma_{+} \sigma_{-}\right)+g\left(a \sigma_{+}+a^{\dagger} \sigma_{-}\right) \\
& -z t \psi\left(a^{\dagger}+a\right)+z t|\psi|^{2},
\end{aligned}
$$

with $\mu, z$, and $t$ the chemical potential, number of nearest neighbors, and hopping frequency, respectively, and $\psi=\langle a\rangle$ the mean field. This Hamiltonian and $H_{\mathrm{JCD}}^{\text {int }}$ do read the same if we identify $\mu-\omega_{0}$ with $\Delta \omega$, zt $\psi$ with $\mathcal{E}$, and add $|\mathcal{E}|^{2} / z t$ to $H_{\mathrm{JCD}}^{\text {int }}$; if we identify $z t$ with $\kappa$, the added term is the drive photon flux and coordinates with $z t|\psi|^{2}$ in Eq. (20). Nevertheless, despite the similarity, the two Hamiltonians are put to very different uses. The drive field $\psi=\langle a\rangle$ in Eq. (20) is a self-consistent field, determined by minimizing the ground-state energy (see, e.g., the Methods section of Ref. [20]). It is the superfluid order parameter, which plotted as a function of $\mu-\omega_{0}$ and $t$ maps out the photonic Mott-insulator-to-superfluid phase diagram. The drive in $H_{\mathrm{JCD}}^{\mathrm{int}}$ is an external field, held fixed as an input, with steady-state quantities like $\langle a\rangle$ or $\left\langle a^{\dagger} a\right\rangle$ determined by a balance between this input and photon loss. An equation like $z t \psi \rightarrow \kappa\langle a\rangle=\mathcal{E}$ does not generally hold. It holds if the dipole coupling is 0 , as the steady state for a coherently driven single cavity or array of cavities is a cavity or cavities in a coherent state of amplitude $\mathcal{E} / \kappa$. On the other hand, for a single driven cavity with fixed input and nonzero dipole coupling, we must solve a master equation to determine the steady state in the presence of fluctuations.

\section{A DISSIPATIVE QUANTUM PHASE TRANSITION}

Alsing and Carmichael [27] solve the Lindblad master equation (in the interaction picture)

$$
\frac{d \rho}{d t}=\frac{1}{i \hbar}\left[H_{\mathrm{JCD}}^{\mathrm{int}}, \rho\right]+\kappa\left(2 a \rho a^{\dagger}-a^{\dagger} a \rho-\rho a^{\dagger} a\right),
$$

with $\rho$ the reduced density operator, to verify the mean-field prediction of Eqs. (14)-(19). Adopting a resonant drive, and dipole-coupling strength $g / \kappa=10$, they plot the $Q$ function in steady state

$$
Q(x+i y)=\left\langle x+i y\left|\rho_{\mathrm{SS}}\right| x+i y\right\rangle .
$$

$|x+i y\rangle$ a coherent state, and they demonstrate how it changes from a single peak below the critical point $2 \mathcal{E} / g=$ 0.96 to a double peak above $2 \mathcal{E} / g=1.04$ (Fig. 2 of Ref. [27]). They report what they call spontaneous dressed-state polarization - phase bistability of the polarization and cavity field for resonant driving above the critical point (see also Ref. [58]).
By mapping out the photon-number mean and region of bimodal $Q$ function in the plane of drive amplitude and detuning, their work is extended in this section to cast the critical point at $\Delta \omega=0$ and $2 \mathcal{E}=g$ as the organizing center of the breakdown of photon blockade. All results are for a dipole-coupling strength $g / \kappa=50$.

\section{A. Mean photon number in steady state}

I numerically solve Eq. (21) using a standard fourthorder Runge-Kutta algorithm. The mean photon number in the steady state is plotted as a function of drive amplitude and detuning in Fig. 1. The three panels on the left show detuning ranges -60 to $60,-16$ to 16 , and -4 to 4 (bottom to top) for corresponding ranges of drive amplitude 0 to 10 , 10 to 20 , and 20 to 30 . Each panel displays contours of the surface plot shown to the right. A driven empty cavity would yield a Lorentzian of half-width $\Delta \omega / \kappa=1$ and a maximum of 900 photons at $\Delta \omega / \kappa=0$ for the largest drive amplitude $\mathcal{E} / \kappa=30$. The most prominent feature of the figure is that the Lorentzian line is split in two, with no significant growth of the on-resonance photon number until the critical point (white dot in the upper contour plot) is reached. The second-order transition at the critical point is also prominent in the view down the channel formed by the Lorentzian line in the surface plots (compare the top and middle plots).

The physical origin of the split Lorentzian is clear from the Jaynes-Cummings Hamiltonian written in diagonal form:

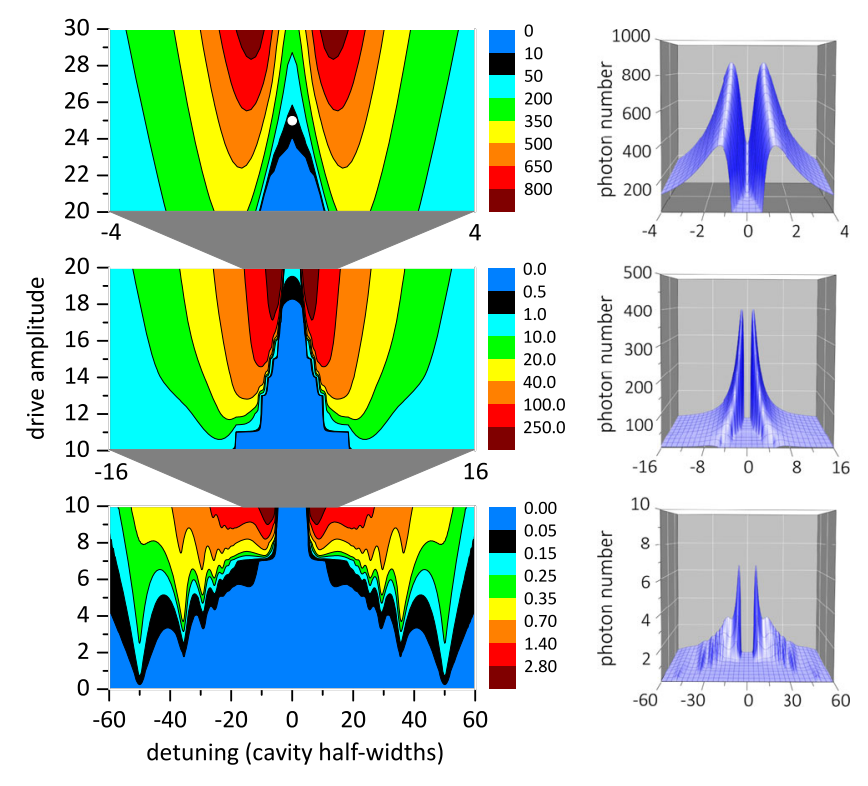

FIG. 1. Mean photon number in the steady state as a function of drive detuning $\Delta \omega / \kappa$ and amplitude $\mathcal{E} / \kappa$ for a dipole-coupling strength $g / \kappa=50$. The white dot in the uppermost panel locates the critical point at $\Delta \omega / \kappa=0$ and $\mathcal{E} / \kappa=25$. Each panel on the left shows contours of the surface plot on the right. 


$$
\begin{aligned}
H_{\mathrm{JC}}= & E_{0}\left|E_{0}\right\rangle\left\langle E_{0}\left|+\sum_{n=1}^{\infty} E_{n, U}\right| E_{n, U}\right\rangle \\
& \times\left\langle E_{n, U}\left|+\sum_{n=1}^{\infty} E_{n, L}\right| E_{n, L}\right\rangle\left\langle E_{n, L}\right|,
\end{aligned}
$$

where $E_{0}=0$ is the ground-state energy, $\left|E_{0}\right\rangle=|0,-\rangle$, and $E_{n,(U, L)}$ and $\left|E_{n,(U, L)}\right\rangle$ are given in Eqs. (3)-(5). In response to the coherent drive, transitions out of the ground state have two available paths of excitation up the JaynesCummings ladder: an upper path and a lower path, which step, respectively, between levels of the anharmonic oscillators defined by the second and third terms of Eq. (23). At large photon number (with strong dipole coupling), the paths are largely independent of one another, as matrix elements for transitions between them are extremely small (see Sec. V). Considering each path separately, the drive detuning at large $n$ is approximated by $E_{n+1,(U, L)}-E_{n,(U, L)}-\hbar \omega_{D} \approx-\hbar \Delta \omega \pm \hbar g / 2 \sqrt{n}$, which yields, balancing the coherent driving against loss,

$$
n=\frac{\mathcal{E}^{2}}{\kappa^{2}+(\Delta \omega \mp g / 2 \sqrt{n})^{2}} .
$$

The simple result is an approximation to Eq. (19), where the minus sign applies to the upper excitation pathbrought to resonance by positive detuning - and the plus sign to the lower-brought to resonance by a negative detuning. The nonlinear dispersion is offset at $\Delta \omega / \kappa=$ $\pm g / 2 \mathcal{E}$ with photon number $n=(\mathcal{E} / \kappa)^{2}$, i.e., the photon number reached in an empty cavity driven on resonance. This resonance condition locates the two peaks of the split Lorentzian in the top panel of Fig. 1.

At the low photon numbers of the bottom panel of Fig. 1, the steps of the Jaynes-Cummings ladder are far too anharmonic for the approximation of Eq. (24) to hold. This is the regime of strong multiphoton blockade; four or five multiphoton resonances are seen in the figure, similar to what is measured in the circuit QED experiment of Bishop et al. [8]. The resonance at $\Delta \omega / \kappa= \pm 50$ corresponds to one-photon blockade-photon blockade as it is usually discussed [13,14,20-22]. Two-, three-, and fourphoton resonances appear for larger drive amplitude and ever smaller detunings; each exhibits a similar multiphoton blockade [26]. The multiphoton resonances merge beyond the fourth or fifth, to ultimately accumulate in a broad blockade region around cavity resonance. The accumulation marks the entrance to a deep channel running between the peaks of the split Lorentzian and leading up to the critical point.

The steep walls of the channel leading to the critical point correspond to a domain of coexistence, a region in the drive-amplitude-detuning plane where the vacuum state and a state of high photon number-with the blockade broken through-coexist [29,30]. They evidence a dissipative quantum phase transition of first order.

\section{B. Bimodality and critical slowing down}

I use bimodality of the steady-state $Q$ function as an indicator of coexistent states. The choice, of course, is not unique; alternatively, the Wigner function, or even the photon-number distribution, might be used. While different measures will not agree in every detail-due, for example, to the larger width of a $Q$ relative to Wigner function-we can nevertheless expect agreement on the main features of the domain of coexistence. Differences will be limited to boundaries where the two peaks of a bimodal distribution coalesce.

The domain of coexistence corresponding to Fig. 1 is mapped out with contours of equal peak-height ratio in Fig. 2(a); examples of bimodal $Q$ functions are shown in Figs. 2(b)-2(e). The bimodal region below the critical point consists of symmetric positive and negative detuning lobes. The brown stripe running through the center of each indicates a peak-height ratio close to one and the black stripe along the edges a ratio close to 0.2. An abrupt transition, from a bimodal to a single-peaked $Q$ function, terminates each lobe at the large detuning end; along this boundary, the two peaks of a bimodal distribution coalesce. The red lines, set apart from the contour plot, mark the boundary of the bistable region predicted on the basis of a mean-field treatment. The domain of coexistent states determined from the master equation is contained within this boundary but because of fluctuations is much reduced. Clearly, fluctuations are important. We return to a comparison between mean-field theory and the full solution including fluctuations in Sec. IV B.

Figure 3 adds detail to what we see in Fig. 2. Figure 3(a) plots the mean photon number (red boxes) as a function of detuning for a drive amplitude $\mathcal{E} / \kappa=18$. The domain of coexistent states is reduced at this drive amplitude to a pair of narrow strips, one on either side of resonance; each strip ranges from approximately $|\Delta \omega| / \kappa=1.0$, where the mean photon number starts increasing from 0 , to $|\Delta \omega| / \kappa=1.5$, where a maximum photon number is reached. The two narrow strips of bimodal states align with the steep walls of the channel leading to the critical point in Fig. 1.

The blue and green triangles in Fig. 3(a) compare the time-dependent approach of the mean photon number to the steady state at the center of the range of bimodality (blue) and outside that range (green); states of the same mean photon number (circled in the figure) are compared. The time axis along the top of the figure runs from 0 up to a very large $8 \times 10^{3}$ cavity lifetimes $\left(2 \kappa t=8 \times 10^{3}\right)$. Clearly, when the $Q$ function is bimodal, there can be a dramatic critical slowing down. The figure shows slowing by a factor of 45 when the blue and green triangles are compared and 1000 against the empty-cavity filling-rate. The evolution reflects a slow breaking through of the photon blockade in a 


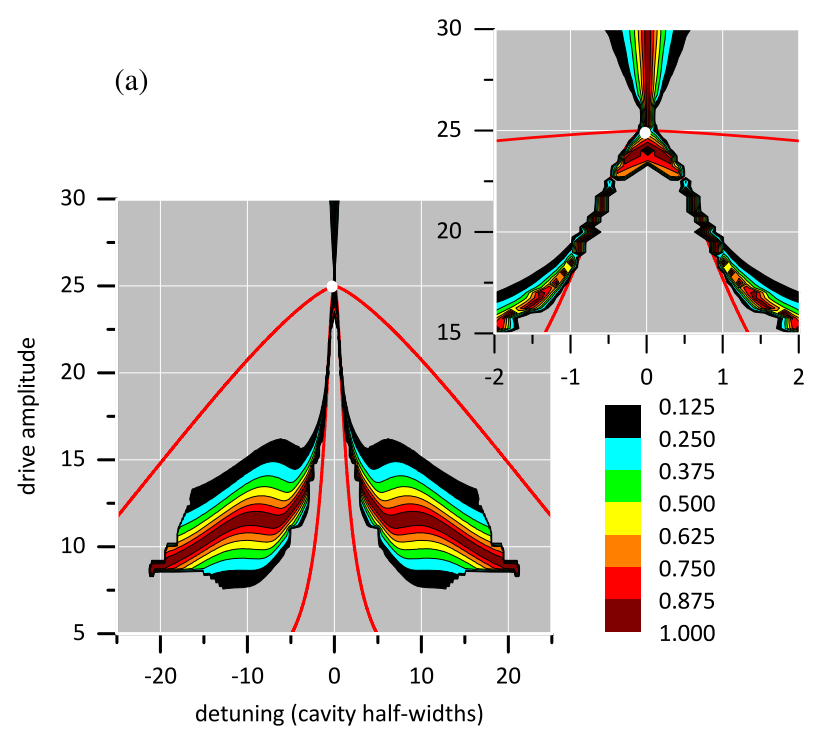

(b)

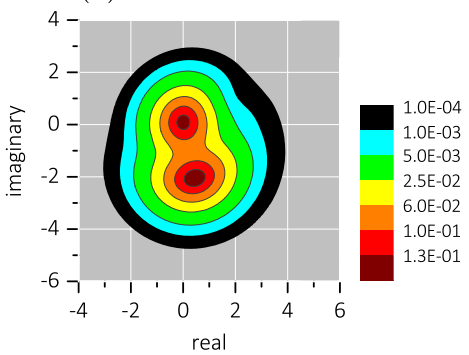

(d)

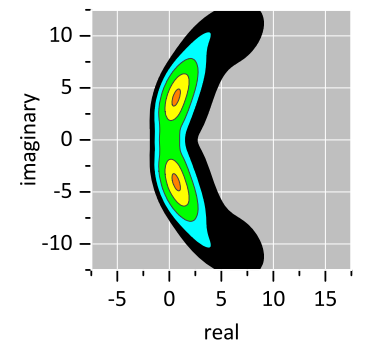

(c)

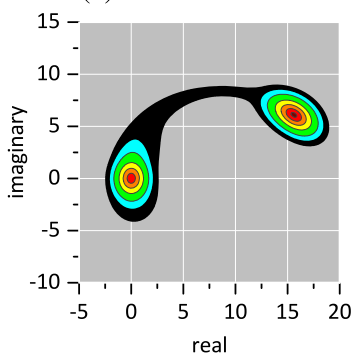

(e)

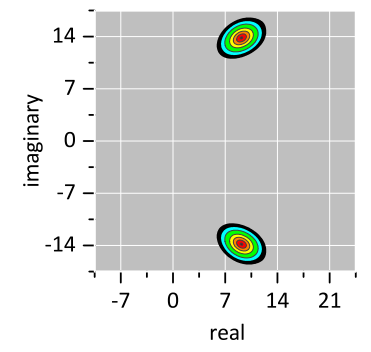

FIG. 2. (a) Region of drive-detuning-amplitude plane within which the steady-state $Q$ function corresponding to Fig. 1 is bimodal. Contours of $r=1-\left|h_{1}-h_{2}\right| /\left(h_{1}+h_{2}\right)$ are shown, with $h_{1}$ and $h_{2}$ the peak heights. Red lines outline the region of bistability according to Eqs. (10)-(12), e.g., as in Fig. 4(b). (b)-(e) Sample $Q$ functions plotted, respectively, for $(\mathcal{E} / \kappa, \Delta \omega / \kappa)=(10,16) ;(18,1.1) ;(25,0) ;(30,0)$.

cascade of multiphoton transitions, with, for drive detuning $|\Delta \omega| / \kappa=1.1$, the photon number taken from 0 to approximately 285 . The coexistent states involved-vacuum state and state of high photon number (mean amplitude $\alpha \approx 15.8+i 6$ ) - are illustrated by the steady-state $Q$ function plotted in Fig. 3(b), where the vertical scale is expanded to reveal the path of excitation (upper half of the contour plot) and deexcitation (lower half of the contour plot). In the displayed case of a positively detuned drive, excitation is via the upper Jaynes-Cummings ladder-

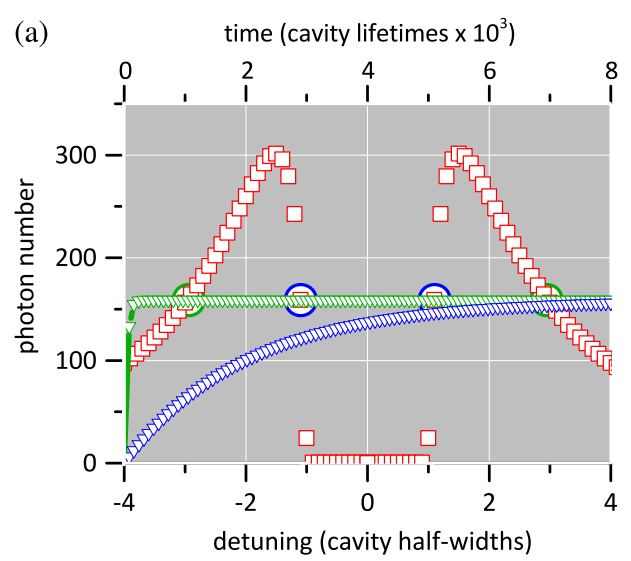

(b)
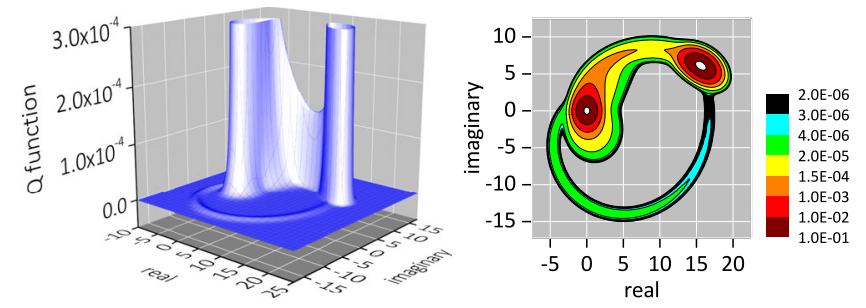

FIG. 3. (a) Mean photon number in steady state versus drive detuning $\Delta \omega / \kappa$ for drive amplitude $\mathcal{E} / \kappa=18$ (red squares). Additional curves show the time evolution to the steady state for $\Delta \omega / \kappa= \pm 1.1$ (blue triangles and blue circles) and $\Delta \omega / \kappa=$ \pm 2.95 (green triangles and green circles); the time axis appears at the top of the graph. (b) Surface plot and contours of the steadystate $Q$ function for $\Delta \omega / \kappa=+1.1$; peak heights are $h_{1}=0.12$ (left peak) and $h_{2}=0.14$ (right peak).

second term on the right-hand side of Eq. (23) — and deexcitation via the lower Jaynes-Cummings ladder-third term on the right-hand side of Eq. (23). We return to these phase-space paths and the fluctuations that follow them in Sec. V.

\section{QUANTUM FLUCTUATIONS AND THE "THERMODYNAMIC LIMIT"}

The critical point was identified in Sec. II B from quantum mechanical quasienergies and from mean-field steady states in Sec. II C. Figures 1 and 2 of Sec. III then confirmed its role as the organizer of behavior in the presence of quantum fluctuations, which neither quasienergies nor mean-field steady states take into account. In this section, we look more carefully at what mean-field theory can tell us and ask, specifically, whether there exists a "thermodynamic limit," a limit of high photon number in which quantum fluctuations vanish. I propose such a limit as a strong-coupling limit, in sharp contrast to previously discussed dissipative phase transitions for photons $[31,32,36,37]$ where the limit of large photon number is a weak-coupling limit. Yet, for the breakdown of photon 
blockade, the vanishing of fluctuations does come with a qualification [below Eq. (35)].

The words "thermodynamic limit" are set in quotes. It is important to distinguish what is said for photons from the standard thermodynamic limit in extended systems (e.g., lattices), where particle number is conserved, fixed a priori, and scales in an obvious way with lattice size or number of degrees of freedom. The common factor is the notion of a large number limit. The question asked of Fig. 1 is what governs the photon-number scale -0 to a maximum 900 photons for the parameters used? The peak photon number in Fig. 3(a), for example, might be 30, 300, or 3000 photons, and the drop in number, sharp, sharper, or sharper still. The sense of moving toward a "limit" captures these trends, even though the strict limit to infinity may not be physically relevant for experiments that respect the assumptions of the model.

In what follows, we recall first how a "thermodynamic limit" for photons, far from thermal equilibrium, may be defined by considering the number of photons required to "activate" a mean-field nonlinearity, in the sense that nonlinear terms cease to be a negligible perturbation. We consider the saturable nonlinearity of Maxwell-Bloch equations, i.e., a nonlinearity produced by saturating a two-level system in the presence of excited-state decay, e.g., spontaneous emission. The example introduces the commonly met weak-coupling "thermodynamic limit" for photons, prior to the strong-coupling limit that holds for the breakdown of photon blockade (Sec. IV C).

\section{A. "Thermodynamic limit" for a saturable transition}

Spontaneous emission to modes of the radiation field other than the cavity mode breaks the conservation law $4|\beta|^{2}+\zeta^{2}=1$; the neoclassical equations are replaced by Maxwell-Bloch equations:

$$
\begin{gathered}
\frac{d \alpha}{d t}=-(\kappa-i \Delta \omega) \alpha-i g \beta-i \mathcal{E}, \\
\frac{d \beta}{d t}=-(\gamma / 2-i \Delta \omega) \beta+i g \alpha \zeta, \\
\frac{d \zeta}{d t}=-\gamma(\zeta+1)+2 i g\left(\alpha^{*} \beta-\alpha \beta^{*}\right),
\end{gathered}
$$

with $\gamma$ the spontaneous emission rate. Steady states of $\alpha$ then satisfy the state equation of optical bistability for a saturable two-state transition [35]:

$$
\alpha=-i \mathcal{E}\left[\kappa-i \Delta \omega+\frac{g^{2}(\gamma / 2+i \Delta \omega)}{\gamma^{2} / 4+\Delta \omega^{2}+2 g^{2}|\alpha|^{2}}\right]^{-1} .
$$

They follow from the response of a Lorentzian resonance both broadened and shifted by saturable absorption and dispersion. Although Eq. (28) is normally written down for a medium of density $N / V$ (with $g^{2} \rightarrow N g^{2}$ in the numerator) [36,59], single-atom optical bistability [60] is the natural extrapolation away from the results reported in Secs. II and III.

On resonance, the nonlinearity of Eq. (28) is brought into play (no longer a negligible perturbation) for photon numbers

$$
|\alpha|^{2} \sim n_{\text {sat }}=\gamma^{2} / 8 g^{2} .
$$

The saturation photon number $n_{\text {sat }}$ may then be read as a typical photon number, and a "thermodynamic limit" $n_{\text {sat }} \rightarrow \infty$ thus defined. The limit arrived at in this way is a weak-coupling limit, since

$$
g^{2}=\frac{\omega_{0} \mu^{2}}{2 \hbar \epsilon_{0} V}
$$

where $\mu$ is the dipole moment, so taking $g^{2}$ to 0 implies that $n_{\text {sat }}$ and $V$ are taken simultaneously to infinity. The limit is, of course, a little artificial when just one two-state system is considered because, without putting $\mathrm{Ng}^{2}$ in the numerator of Eq. (28), the nonlinear polarization vanishes altogether if $g^{2} \rightarrow 0$. The more natural limit is $g^{2} \rightarrow 0, N \rightarrow \infty$ with the cooperativity parameter $C=N g^{2} / \gamma \kappa$ (density $N / V$ ) fixed [36,59]. Nonetheless, a meaningful limit can be defined for $N=1$ by letting $g^{2} \rightarrow 0, \kappa \rightarrow 0$ with $C_{1}=g^{2} / \gamma \kappa$ fixedor, more correctly, $(g / \gamma)^{2} \rightarrow 0,(\kappa / \gamma) \rightarrow 0$ with $C_{1}$ fixed. We will return to results moving in this direction in Sec. V.

\section{B. Comparison with mean-field theory}

Although the neoclassical equations are Maxwell-Bloch equations with the spontaneous emission rate $\gamma$ set to 0 , their steady-state solution is not recovered quite so directly: setting $\gamma$ to 0 in Eq. (28) does not return Eq. (19). Since the Maxwell-Bloch equations break the conservation law $\zeta^{2}+4|\beta|^{2}=1$, they reach a steady state in the interior of the Bloch sphere rather than on its surface; indeed, the onresonance steady state moves to the center of the sphere as $\gamma$ goes to 0 . There is, however, a special case. In the presence of weak nonlinear dispersion,

$$
2 g^{2}|\alpha|^{2} \ll \Delta \omega^{2},
$$

Eqs. (19) and (28) (with $\gamma=0$ ) agree — both yield the state equation for a Kerr nonlinearity to lowest order in $2 g^{2}|\alpha|^{2} / \Delta \omega^{2}$ (see, e.g., Sec. VII 5. of Ref. [59]):

$$
\alpha=-i \mathcal{E}\left\{\kappa-i\left[\Delta \omega-\frac{g^{2}}{\Delta \omega}\left(1-\frac{2 g^{2}}{\Delta \omega^{2}}|\alpha|^{2}\right)\right]\right\}^{-1} .
$$

Note that the solution with $\zeta<0$ is chosen to arrive at Eq. (32) from Eq. (19) (the other solution is unstable). Considering linear dispersion only, i.e., when the Kerr nonlinearity is negligible, this equation returns vacuum Rabi resonances at $\Delta \omega= \pm g$, in the manner of the 
treatment of vacuum Rabi splitting made by Zhu et al. [61]. The Kerr nonlinearity pulls the vacuum Rabi resonances toward line center, eventually inducing a large enough tilt to recast each as a bistable "switch"—-so each vacuum Rabi resonance exhibits hysteresis as the drive detuning is scanned [62].

Figure 4(a) shows an example of dispersive bistability like this, where $|\alpha|^{2}$ - the mean-field photon number-is plotted from Eq. (19) for a drive amplitude a little beyond the regime where Eq. (32) would hold. The photon number is everywhere less than 1 , a sure sign that the full quantum treatment cannot agree with the mean-field prediction. Gray curves compare the quantum result at three different drive amplitudes within the range of the lower panel of Fig. 1; the smallest of the three corresponds to the mean-field plot. The departure from mean-field theory is clear. The photon blockade remains in place. We observe only a partially saturated vacuum Rabi resonance, where in the two-state approximation, each vacuum Rabi resonance saturates with peak photon number $n=0.25$ [14]; the peak number is $n \approx 0.2$ for $\mathcal{E} / \kappa=1$ in the figure.

The gray curves for $\mathcal{E} / \kappa=5$ and $\mathcal{E} / \kappa=8$ illustrate the continuing disagreement between the full quantum treatment and mean-field theory. They confirm that the breakdown of photon blockade sets in, not as a dispersive bistability, as mean-field theory predicts, but through a cascade of multiphoton transitions. The expectation that a nonlinearity is "turned on" only by high photon numbers

(a)

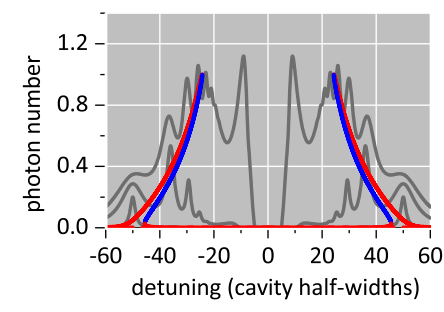

(c)

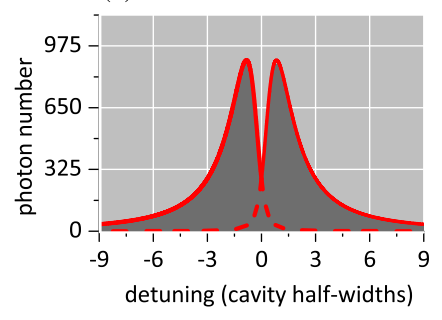

FIG. 4. Mean-field photon number $|\alpha|^{2}$ (red and blue curves), compared with the photon-number mean, including quantum fluctuations (gray), for a dipole-coupling strength $g / \kappa=50$ and drive amplitudes (a) $\mathcal{E} / \kappa=1$ (red and blue) and 1,5,8 (gray, lower to upper), (b) $\mathcal{E} / \kappa=12$, and (c) $\mathcal{E} / \kappa=30$. Steady states plotted as solid red (blue) curves are stable (unstable); those plotted as dashed red curves-running close to $|\alpha|^{2}=0$ in (a) and (b) - have $\zeta>0$ and are unstable. (as in Sec. IV A) holds true for a perturbation away from a linear response, e.g., in the Kerr nonlinearity of Eq. (32). The present case is different, as the nonlinearity of Eq. (19) diverges on resonance as the photon number goes to $0-\mathrm{a}$ clear sign that mean-field theory fails and quantized energy levels are needed.

Note that the divergence is encountered only when the two-state system and field mode are resonant with one another. Detuning "softens" the nonlinearity, and in the strong dispersive regime, where the detuning $\delta$ is much larger than the coupling strength [63], Eq. (19) is replaced by the saturable extension of the Kerr model

$$
\alpha=-i \mathcal{E}\left\{\kappa-i\left[\Delta \omega-\frac{g^{2}}{\delta}\left(1+\frac{4 g^{2}}{\delta^{2}}|\alpha|^{2}\right)^{-1 / 2}\right]\right\}^{-1},
$$

where, since $g^{2} / \delta^{2} \ll 1$, we return to a nonlinearity that is "turned on" only by high photon numbers, essentially restoring the mean-field bistability.

At higher drive amplitudes, in particular, above the critical point, mean-field theory and the full quantum treatment are brought into close agreement. Figure 4(c) makes the comparison for $\mathcal{E} / \kappa=30$ (top of the upper panel in Fig. 1); the agreement is not exact, but the small difference is not apparent at the resolution of the figure.

The region of coexistent states (Fig. 2) connects these two extremes, where, at intermediate drive strengths, the mean-field prediction of dispersive bistability supports the proposal of a first-order transition running along the walls of the channel leading to the critical point in Fig. 1. Figure 4(b) compares mean-field steady states (red and blue curves) with the photon-number mean (gray shading) for $\mathcal{E} / \kappa=12$ (lower half of the middle panel in Fig. 1). Of course, fluctuations are an essential part of the story, making the connection between these results. The photon-number mean is to be understood as an average over spontaneous switching between the mean-field steady states.

I illustrate the influence of quantum fluctuations with the aid of quantum trajectory simulations in Fig. 5. To arrive at the yellow and green curves, the photon-number expectation is plotted against $\Delta \omega(t) / \kappa$ while the drive detuning is scanned with the drive amplitude fixed; the result is superposed on the steady-state plot of Fig. 4(b). The scan of Fig. 5(a) is "fast," with a scan rate of $0.1 \kappa$ per $\kappa^{-1}$. It shows dynamical symmetry breaking: With $\Delta \omega / \kappa$ changing from negative to positive (yellow curve), the photon number climbs via excitation of the lower JaynesCummings oscillator-third term on the right-hand side of Eq. (23) - while with $\Delta \omega / \kappa$ changing from positive to negative (green curve), it climbs the ladder of the upper Jaynes-Cummings oscillator-second term on the righthand side of Eq. (23). There is a noisy period preceding each deexcitation (precipitous drop to 0), but there is no "blinking" of the photon number on and off. Figure 5(b) 
(a)

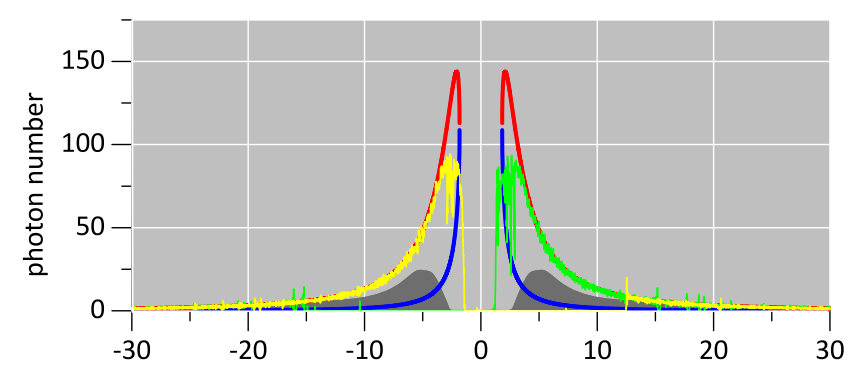

(b)

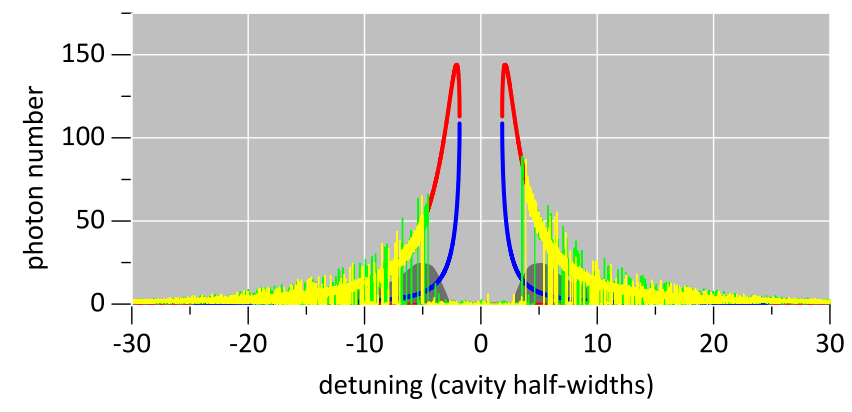

FIG. 5. Sample quantum trajectories show dynamical symmetry breaking as the drive detuning $\Delta \omega / \kappa$ is scanned. Time-dependent photon-number expectations are superposed on the plot of Fig. 4(b), for scan times (one direction) of (a) $\kappa T=6 \times 10^{2}$ and (b) $\kappa T=6 \times 10^{4}$. Yellow (green) curves plot the scan from left to right (right to left).

shows how symmetry is recovered in a "slow" scan, with a scan rate of $0.001 \kappa$ per $\kappa^{-1}$. Here, the photon number does "blink" on and off, thus restoring symmetry with respect to scan direction; a time average at fixed detuning recovers the photon-number mean (gray shading) from quantum trajectories that switch back and forth between mean-field steady states.

\section{C. "Thermodynamic limit"}

Returning to the discussion of Sec. IVA, the photonnumber scaling of the mean-field state equation [Eq. (19)] shows exactly the reverse dependence on dipole-coupling strength to the saturable nonlinearity of Eq. (28). The mean-field photon number satisfies (with $\zeta<0$ )

$\frac{|\alpha|^{2}}{n_{\text {scale }}}=\left(\frac{2 \mathcal{E}}{g}\right)^{2}\left[1+\left(\frac{\Delta \omega}{\kappa}-\frac{1}{\sqrt{\Delta \omega^{2} \kappa^{2} / g^{4}+|\alpha|^{2} / n_{\text {scale }}}}\right)^{2}\right]^{-1}$

where $n_{\text {scale }}=g^{2} / 4 \kappa^{2}$; on resonance,

$$
\left(|\alpha|^{2} / n_{\text {scale }}\right)\left[\left(|\alpha|^{2} / n_{\text {scale }}\right)+1-(2 \mathcal{E} / g)^{2}\right]=0 .
$$

The "thermodynamic limit" takes $n_{\text {scale }}$ to infinity. It is a strong-coupling limit, in sharp contrast to the limit of Sec. IV A. Is it, however, a limit in which fluctuations vanish? We should consider that while photon numbers scale with $n_{\text {scale }}$ above and near the critical point, the nonlinearity of Eq. (34) diverges (on resonance) as $|\alpha|^{2}$ goes to 0. Energy-level shifts due to one, two, three, etc., photons are fundamental to photon blockade (lower panel of Fig. 1) with associated multiphoton resonances that are missed by the mean-field nonlinearity of Eq. (34) [Fig. 4(a)]. Can something be said about the role of fluctuations in this regime?

We focus on the lower panel of Fig. 1 and low drive amplitudes in Fig. 2, where mean-field theory and the full quantum treatment disagree. The following argument, if a little crude, suggests that this regime of disagreement persists-i.e., does not collapse to $n / n_{\text {scale }}=0$ - as $n_{\text {scale }}$ is taken to infinity. I argue from the detuning of the $n$-photon resonance away from the blockaded next step - the $n$ plus first step —up the Jaynes-Cummings ladder: with $n \hbar \omega_{D}=$ $n \hbar \omega_{0} \mp \sqrt{n} \hbar g$ and $n$ large,

$$
E_{n+1,(U, L)}-E_{n,(U, L)}-\hbar \omega_{D} \approx \mp \sqrt{\frac{n_{\mathrm{scale}}}{n}} \hbar \kappa .
$$

Assume then that the $n$-photon blockade persists up to a minimum detuning of $m \hbar \kappa$, i.e., up to some $n_{\max }$ with $\sqrt{n_{\text {scale }} / n_{\max }} \sim m$. Assume also that the $n_{\max }$-photon transition is saturated;i.e., the mean photon number is $n_{\max } / 4$. We thus arrive at a bound $n_{\max } / 4 \sim n_{\text {scale }} / 4 m^{2}$ on the photon number in the regime of multiphoton blockade. Although crude, with $m=10$, the bound gives reasonable numbers, $n_{\max } \sim 6$ and $n_{\max } / 4=1.5$, for $n_{\text {scale }}=625$ as in Fig. 1 . I suggest, furthermore, that the regime of multiphoton blockade grows linearly with $n_{\text {scale }}$ and therefore does not collapse to $n / n_{\text {scale }}=0$ in the "thermodynamic limit."

Of course, quantum fluctuations determine the drive detuning that locates the first-order transition along the channel leading up to the critical point in Figs. 1 and 2, e.g., in Fig. 3(a) around $\Delta \omega / \kappa= \pm 1.1$. An equilibrium transition is located according to the Maxwell equal-area construction, but the construction does not carry over to the nonequilibrium case, where a generalization that balances fluctuations in the presence of nonlinearity holds $[64,65]$. The brown stripe $(r \approx 1)$ through the domain of coexistence in Fig. 2 should approximate the location of this transition, although its point of termination, at $|\Delta \omega / \kappa| \approx 20$ and $\mathcal{E} / \kappa \approx 9$, likely depends on the chosen criterion for coexistence (bimodality of the $Q$ function) and finite value of $n_{\text {scale }}=625$.

\section{LADDER SWITCHING THROUGH SPONTANEOUS EMISSION}

In contrast to quantum phase transitions in thermal equilibrium, dissipative quantum phase transitions are influenced in a fundamental way by their inputs and outputs. Outputs send information into the environment, 
which might potentially identify - in effect, "measure"the open-system quantum state, while inputs impose boundary constraints, e.g., an imposed rather than selfconsistent drive (Sec. II D). The behavior of Secs. II-IV is formed around the cavity input and output: Driven off resonance, the nonlinear response is drawn from either the upper or lower Jaynes-Cummings ladder, whether upper or lower imposed by the drive detuning; neither ladder is drive selected on resonance-a parity-breaking decision is made against a background of fluctuations, the result revealed by information in the environment, i.e., by measuring the output field.

Spontaneous emission provides a second channel for leaking information to the environment. In this section, I show how spontaneous emission connects the strongcoupling "thermodynamic limit" (Sec. IV C) to the weakcoupling limit (Sec. IVA). I show how a spontaneous emission quantum jump triggers an environment-induced measurement revealing the Jaynes-Cummings ladder in play-through a 50/50 selection, spontaneous emission quantum jumps induce ladder switching. A high rate of switching destroys the split-line structure built around broken parity states, transforming it into the nonlinear response of a saturable transition.

The excited-state doublets of the Jaynes-Cummings Hamiltonian entangle photon number with "up" and "down" states of the two-state system; they are $n$-quanta cavity polaritons. The split line of Fig. 1 distinguishes $U$ - from $L$-polariton states. It is important in this regard that the $\{U, L\}$ identity be preserved by the quantum jump under cavity loss-at least to good approximation. Although the jump most generally mixes $U$ and $L$,

$$
\begin{aligned}
a\left|E_{n,\{U, L\}}\right\rangle= & \frac{\sqrt{n}+\sqrt{n-1}}{2}\left|E_{n-1,\{U, L\}}\right\rangle \\
& +\frac{\sqrt{n}-\sqrt{n-1}}{2}\left|E_{n-1,\{L, U\}}\right\rangle,
\end{aligned}
$$

at large photon number, the matrix element for ladder switching is small; the $\{U, L\}$ identity is, indeed, to good approximation, preserved. Rare switches nonetheless do occur. They initiate the "off" stages of the on-off "blinking" of Fig. 5(b). The details are uncovered by Fig. 3(b), where the excitation path from the blockaded vacuum to a state of high photon number is the broad path between the peaks in the contour plot (high "skirt" joining the peaks in the surface plot) and deexcitation follows the narrow path in the contour plot (low "ripple" connecting the peaks in the surface plot). Although not so clear in Fig. 3, the blink-off path (deexcitation) in fact follows a spiral, wrapping around and terminating at the origin [see Fig. 6(b)]. It is the path of free decay down the Jaynes-Cummings ladder that is not quasiresonant with the drive [lower ladder for Fig. 3(b)] and reached from the quasiresonant (upper) ladder through a rare ladder switching event.

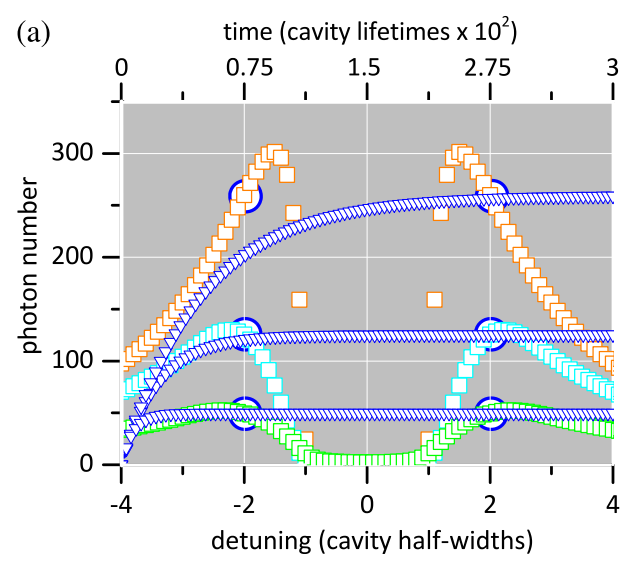

(b)
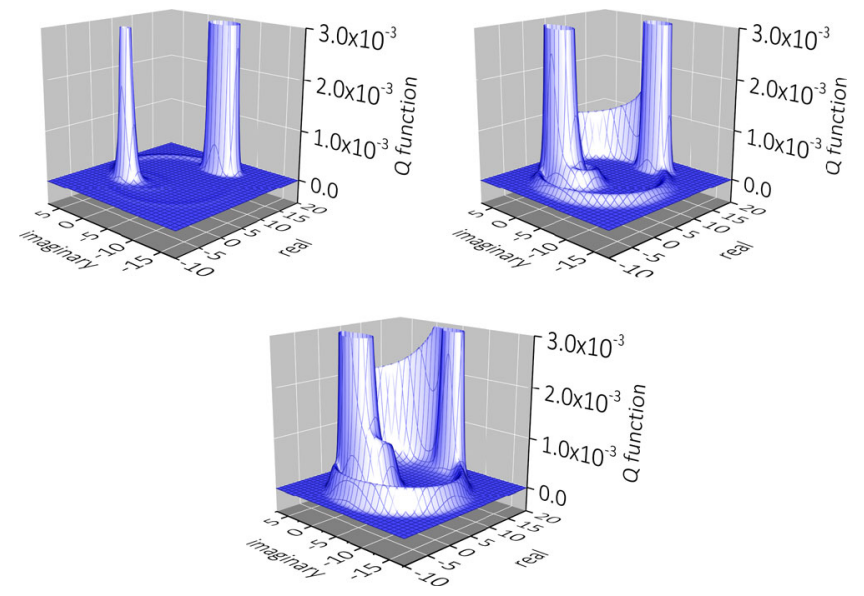

FIG. 6. (a) Mean photon number in the steady state versus detuning $\Delta \omega / \kappa$ for drive amplitude $\mathcal{E} / \kappa=18$ in Fig. 1 and spontaneous emission rate $\gamma / \kappa=0$ (orange), 0.1 (cyan), and 1 (green). Additional curves (blue triangles) show the time evolution to steady state (blue circles) for $\Delta \omega / \kappa= \pm 2.0$; the time axis appears at the top of the graph. (b) Surface plot of the steady-state $Q$ function for $\gamma / \kappa=0$ (upper left), 0.1 (upper right), and 1.0 (lower); left peak heights $h_{l}=0.006,0.14,0.19$; right peak heights $h_{r}=0.28,0.12,0.02$.

Spontaneous emission is much more likely to initiate ladder switching than cavity loss. The quantum jump mixes $U$ and $L$ in equal proportion, where, setting aside an overall phase,

$$
\sigma_{-}\left|E_{n,\{U, L\}}\right\rangle=\frac{1}{2}\left|E_{n, U}\right\rangle+\frac{1}{2}\left|E_{n, L}\right\rangle
$$

Subsequently, environment-induced measurement by the cavity output resolves the superposition, yielding a 50/50 chance of a ladder switch. With the drive on resonance and above the critical point, ladder switching induced by spontaneous emission is read as phase switching of the output field; such phase switching serves as a key indicator of spontaneous dressed-state polarization (see Sec. 5 of Ref. [27] and also Ref. [58] and Sec. 10.5 of Ref. [66]). It 
has been experimentally observed in a high-bandwidth homodyne measurement of the light transmitted by a Fabry-Perot traversed by a cloud of laser-cooled ${ }^{133} \mathrm{Cs}$ atoms [67].

With the drive off resonance, Fig. 6 illustrates the effect of spontaneous emission below the critical point, where the superposition [Eq. (38)] is resolved by output field intensity rather than phase: Does the output remain "bright"-no ladder switch-or does it blink "off" in a switch to the ladder not quasiresonant with the drive? Spontaneous emission shortens the wait to the next "off" switch, in this way driving the on-off "blinking" at a faster rate. Figure 6(a) shows how this erodes the walls of the deep channel leading up to the critical point in Fig. 1, and how the time to the steady state is reduced (blue triangles in the figure). The faster "blinking" is also evident from the increasingly prominent pathways for excitation and deexcitation shown by the series of phase-space plots in (a)

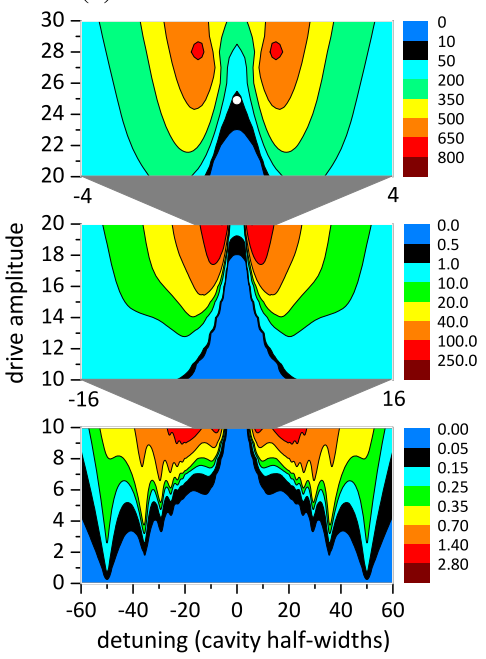

(c)

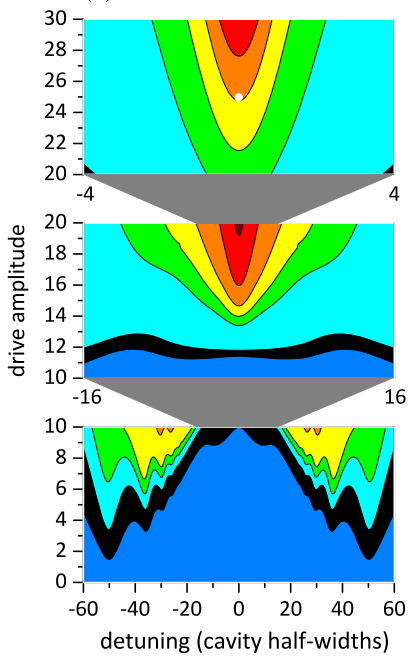

(b)

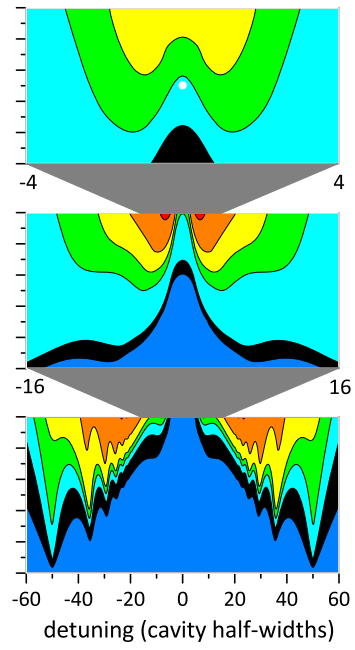

(d)
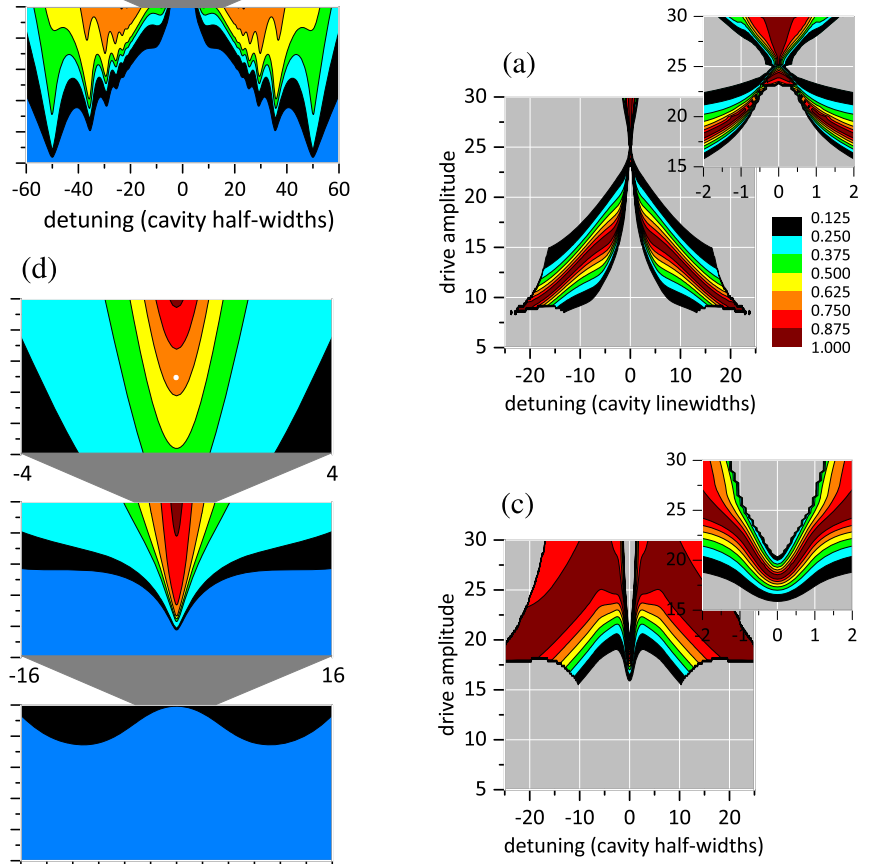

Fig. 6(b) - that the "blinking" is driven specifically by the "off" switch is clear from the changing ratio of peak heights: $h_{l} / h_{r}=0.02,1.2,9.5$ for $\gamma / \kappa=0,0.1,1.0$, where $h_{l}$ refers to the vacuum peak and $h_{r}$ to the "bright" state ( $n=276$ photons in the cavity). Note the spiraling free decay down the detuned Jaynes-Cummings ladder following an excited ladder switch.

The spontaneous emission rates of Fig. 6 correspond to saturation photon numbers of $n_{\text {sat }}=0,5 \times 10^{-7}$, and $5 \times 10^{-5}$, far from the "thermodynamic limit" of Sec. IV A. I present results illustrating the link to this limit in Figs. 7 and 8, where spontaneous emission rates 10 and 100 times larger are added-saturation photon numbers $n_{\text {sat }}=0.005$ and $n_{\text {sat }}=0.5$; although $n_{\text {sat }}=0.5$ might still seem very small, it is large enough to illustrate the link brought by spontaneous emission between the weak-coupling limit of Sec. IVA and the strong-coupling limit of Sec. IV C. Frames (a) and (b) of Figs. 7 and $8\left(n_{\text {sat }}=5 \times 10^{-7}\right.$ and $n_{\text {sat }}=5 \times 10^{-5}$ ) are changed quantitatively from Figs. 1 and 2 , but they still show a breakdown of photon blockade through a cascade of multiphoton transitions and splitting of the cavity line. Mean photon numbers are lowered and the first-order transition "softened" [Fig. 6(a)], but the landscape overall remains unchanged. Moving to frames (c) and (d) of Figs. 7 and $8\left(n_{\text {sat }}=0.005\right.$ and $\left.n_{\text {sat }}=0.5\right)$, a qualitative change sets in. While multiphoton resonances persist in Fig. 7(c)—though noticeably broadened-there is no longer a splitting of the cavity line; in Fig. 7(d), the resonances, too, are gone.
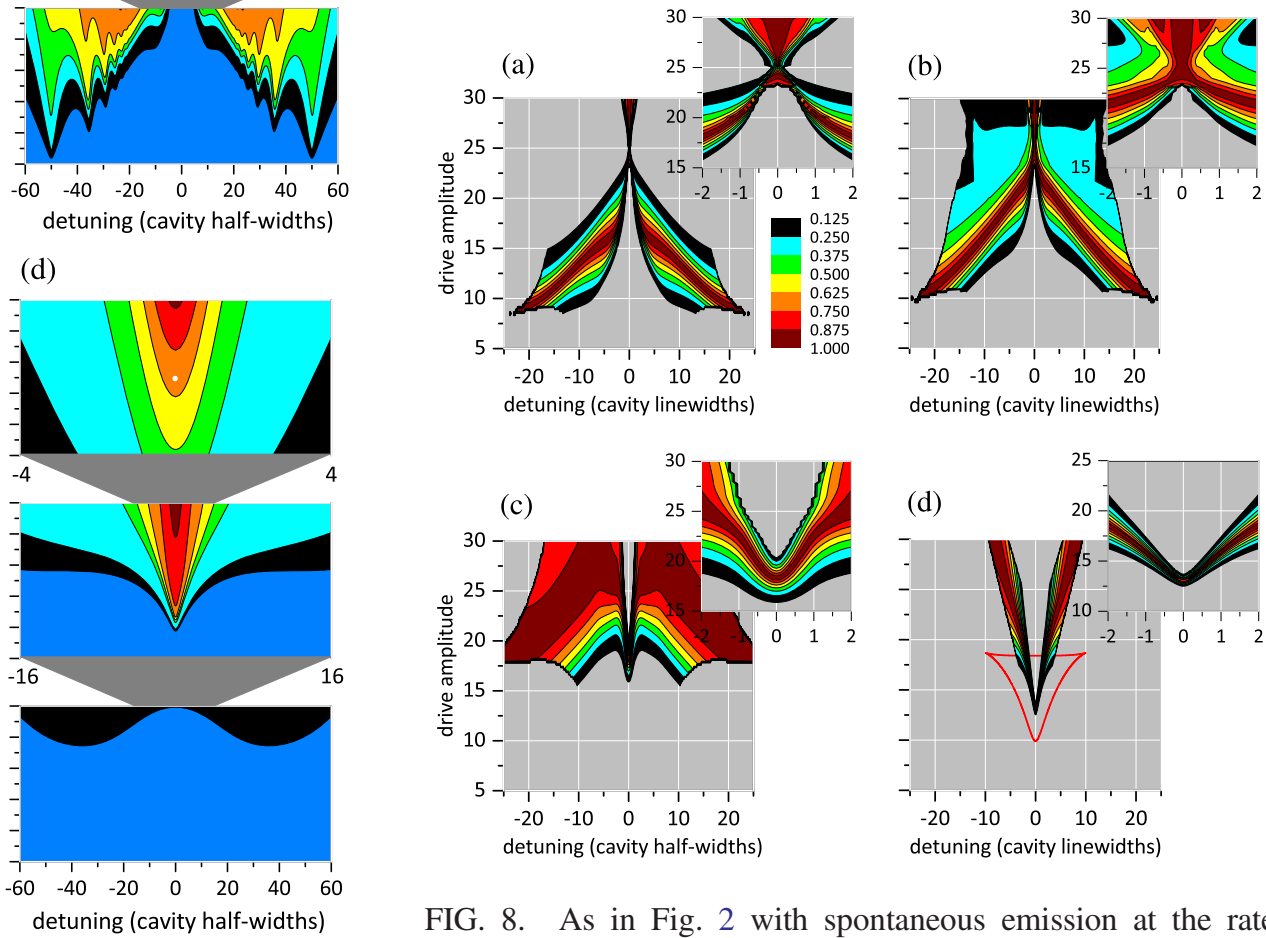

FIG. 7. As in Fig. 1 with spontaneous emission at the rate (a) $\gamma / \kappa=0.1$, (b) $\gamma / \kappa=1$, (c) $\gamma / \kappa=10$, (d) $\gamma / \kappa=100$.
FIG. 8. As in Fig. 2 with spontaneous emission at the rate (a) $\gamma / \kappa=0.1$, (b) $\gamma / \kappa=1$, (c) $\gamma / \kappa=10$, (d) $\gamma / \kappa=100$. The red lines in $(d)$ outline the bistable region according to the state equation [Eq. (28)]. 
The contrasting "thermodynamic limits" of Secs. IV A and IV C are perhaps most apparent from the regions of mean-field bistability, determined, respectively, from the state equation of optical bistability [Eq. (28)] and the neoclassical equation [Eq. (19)]. These regions are outlined with boundaries drawn in red in Figs. 8(d) and 2. The overlayed contours show how the domain of coexistent states-domain of bimodal steady-state $Q$ functionsmaps onto the mean-field prediction, suggesting a Maxwell-like construction. In the case of Fig. 8(d), in particular, although $n_{\text {sat }}=0.5$ is still very far from the "thermodynamic limit" $\left(n_{\text {sat }} \rightarrow \infty\right)$, the emerging construction is similar to that reached by Bonifacio et al. (see Fig. 4 of Ref. [64]) from the potential solution to a small-noise $\left(n_{\text {sat }} \gg 1\right)$ Fokker-Planck equation. The trailing tail at high drive amplitude is an artifact: It comes from $Q$ functions with two local maxima and a local minimum of nearly equal height-i.e., it does not correspond to $Q$ functions with two resolved peaks.

\section{CONCLUSION}

Introduced as an analogue of Coulomb blockade for quantum-well electrons [13] and a signature of strongcoupling cavity QED [14], photon blockade is the key element of proposed photonic extensions of many-body physics models. It is proposed, for example, as a route to a photonic analogue of the superfluid-to-Mott-insulator quantum phase transition of the Bose-Hubbard model [20-22]; although, photon numbers go to 0 at $T=0$ in thermal equilibrium, so experiments on quantum phase transitions for photons employ a laser drive to offset the unavoidable photon loss [46,47]. Inevitably, the photonic extrapolation moves toward dissipative quantum phase transitions, where inputs balance outputs far away from thermal equilibrium (e.g., the transition through laser threshold [31-33]).

I have taken a small step in this direction, exploring the far-from-equilibrium steady states of the Jaynes-Cummings system with a coherent drive and photon loss. I mapped out the mean photon number as a function of drive amplitude and detuning, to show that a previously identified critical point of the driven Jaynes-Cummings Hamiltonian [28] is the organizing center both of strong-coupling dispersive bistabilities [29,30] — coexistence of the blockaded vacuum state and a state of the cavity filled up with light—and a previously reported parity-breaking transition [27]. The photon-number landscape (Figs. 1 and 2) tracks the breakdown of photon blockade, passing, from low photon number, through the saturation of a series of multiphoton resonances, to a phase transition of either second order (on resonance as a function of drive amplitude) or first order (fixed amplitude as a function of detuning).

I also explored the idea of a "thermodynamic limit" in a photonic system driven far from thermal equilibrium, where photon numbers are set by an interplay of nonlinearity and photon loss. I noted how the $\sqrt{n}$ nonlinearity of the Jaynes-Cummings model weakens with increasing photon number, which translates as a divergence of the mean-field nonlinearity as the photon number goes to 0 ; thus, the breakdown of photon blockade acquires a strongcoupling "thermodynamic limit," in contrast to the weakcoupling limit of other dissipative quantum phase transitions for photons-where many photons are required to "turn on" a nonlinearity (see, e.g., Ref. [34]). An example of the latter is the optical bistability studied in the 1980s [35-38]. Finally, I showed how the breakdown of photon blockade is transformed into absorptive optical bistability by dressed-state ladder switching, each switch triggered by spontaneous emission (Figs. 7 and 8).

I treated just one Jaynes-Cummings system - a model in zero dimensions: What can be said about lattices or photonic cavity arrays? I mapped the Jaynes-Cummings Hamiltonian with coherent drive to the effective single-site Hamiltonian of a Bose-Hubbard model in the mean-field decoupling approximation, and the bottom panel of Fig. 1 shows marked similarities to the phase diagram of the Mottinsulator-to-superfluid phase transition (e.g., Fig. 4 of Ref. [20]). "Similar" should not be read, however, beyond what follows from a common level structure: The mean field of the Bose-Hubbard model is a self-consistent field, the coherent drive of the Jaynes-Cummings model an imposed constraint. Although the resonances of Fig. 1 resemble Mott phases of exactly one, two, three, etc., photons per site, they do not mark phase boundaries. They indicate a flow of energy, from input to output, in clumps of one, two, three, etc., photons at a time [26].

Le Boité, Orso, and Cuiti recently applied the mean-field decoupling approximation to a dissipative photonic lattice with coherent drive $[68,69]$. They considered, in particular, an on-site Kerr linearity, where an interaction energy proportional to the square of the photon number provides dispersion proportional to photon number [e.g., as in Eq. (32)]. This model was solved in zero dimensions in the early 1980s [65]. By working in the generalized $P$ representation of Drummond and Gardiner [70], the mean field is found as an explicit function of parameters: In current notation, $\langle a\rangle=A(\mathcal{E} / \kappa, \Delta \omega / \kappa, g / \kappa)$, where $A$ is a ratio of Gamma functions multiplied by generalized hypergeometric functions [65]. Le Boité et al. extend this result to lattices by introducing an effective drive field $\mathcal{E} \rightarrow \mathcal{E}-z t\langle a\rangle$, with $z t$ a nearest-neighbor tunneling rate, and solving

$$
\langle a\rangle=A[(\mathcal{E}-z t\langle a\rangle) / \kappa, \Delta \omega / \kappa, g / \kappa]
$$

self-consistently for $\langle a\rangle$. As the authors point out, their approach differs from a Gross-Pitaevskii approximation, which simply replaces $\mathcal{E}$ by $\mathcal{E}-z t \alpha$ in the Kerr model equivalent of Eq. (10).

The approach might be applied in a similar way to the driven Jaynes-Cummings model. While, in this case, there 
is no known explicit expression $A(\mathcal{E} / \kappa, \Delta \omega / \kappa, g / \kappa)$, the right-hand side of Eq. (39) can always be computed numerically - as the photon number is in Fig. 1. There is reason, however, to think the mean-field decoupling approximation a poor one for treating the breakdown of photon blockade. The authors of Ref. [69] say it is "not fully controlled" and that work beyond the mean field will ultimately determine its domain of validity. Perhaps it can "set" the resonances of Fig. 1 as Mott-like phases, but the transition to high photon number presents a very different challenge.

An approximation of the kind used in Eq. (39) faces two main troubles: It reads a dynamical, or statistical, nonlinearity as a fundamental nonlinearity of interaction, and it neglects site-to-site correlations that clearly follow when the statistical dynamic is "unwound." The point is illustrated by Fig. 5, where, although the photon number is plotted there, what follows holds in an analogous way for the field.

The gray shaded region in Fig. 5 follows the response of the mean photon number as a function of the detuning $\Delta \omega / \kappa$; it is a cut through a function $N(\mathcal{E} / \kappa, \Delta \omega / \kappa, g / \kappa)$, at $\mathcal{E} / \kappa=12$ and $g / \kappa=50$. Importantly, the response is nonlinear, but the nonlinearity is different in kind to what one reads from a mean-field state equation like Eq. (19): It folds in an average over fluctuations [the switching of Fig. 5(b)] assumed to be negligible in a normal mean-field approach. With this average folded into the nonlinearity, it seems dubious that mean fields can be related by an equation like Eq. (39). Alternatively, approaching from the other direction, if we say the fluctuations are present, i.e., the blockade turns on and off, then surely site-to-site correlations must be taken into account.

The breakdown of photon blockade takes the JaynesCummings system from a quantum domain dominated by multiphoton resonance [Fig. 4(a)] to an essentially classical level of high excitation [Fig. 4(c)]. If we can set aside the Mott-insulator-to-superfluid transition per se, the extension to lattices might more convincingly begin with standard many-site mean-field equations, extending Eqs. (10)-(12). A formulation like that of Ref. [65] might then add quantum noise, replacing mean-field equations with stochastic differential equations that respect site-to-site correlations and are suited to numerical simulation. In view of the difficulties faced by simulations within the generalized $P$ representations (see, e.g., Ref. [71]), however, it is far from clear that a program in this direction could be carried through.

A theoretical approach able to follow a transition from one or two photons per site through hundreds to thousands of photons per site across a driven, dissipative lattice is a significant and unmet challenge. Experiments to realize the breakdown of photon blockade in zero dimensions are feasible, on the other hand. Indeed, adding to the one previous experiment with strong coherent drive [67], very recently, a coherent quantum-to-Glauber-state transition for a two-level system coupled to a microwave resonator has been experimentally observed [72].

\section{ACKNOWLEDGMENTS}

This work was supported by the Marsden fund of the RSNZ. Computations were carried out on the NeSI Pan Cluster at the University of Auckland, supported by the Center for eResearch, University of Auckland. Support during two extended visits to the JQI, University of Maryland and NIST, is gratefully acknowledged.

[1] E. T. Jaynes and F. W. Cummings, Comparison of Quantum and Semiclassical Radiation Theories with Application to the Beam Maser, Proc. IEEE 51, 89 (1963).

[2] H. Paul, Induzierte Emission bei starker Einstrahlung, Ann. Phys. (Berlin) 466, 411 (1963).

[3] G. Rempe, H. Walther, and N. Klein, Observation of Quantum Collapse and Revival in a One-Atom Maser, Phys. Rev. Lett. 58, 353 (1987).

[4] M. Brune, F. Schmidt-Kaler, A. Maali, J. Dreyer, E. Hagley, J. M. Raimond, and S. Haroche, Quantum Rabi Oscillation: A Direct Test of Field Quantization in a Cavity, Phys. Rev. Lett. 76, 1800 (1996).

[5] I. Schuster, A. Kubanek, A. Fuhrmanek, T. Puppe, P. W. H. Pinske, K. Murr, and G. Rempe, Nonlinear Spectroscopy of Photons Bound to One Atom, Nat. Phys. 4, 382 (2008).

[6] J. M. Fink, M. Göppl, M. Baur, R. Bianchetti, P. J. Leek, A. Blais, and A. Wallraff, Climbing the Jaynes-Cummings Ladder and Observing its Nonlinearity in a Cavity QED System, Nature (London) 454, 315 (2008).

[7] M. Hofheintz, E. M. Weig, M. Ansmann, R. C. Bialczak, E. Lucero, M. Neeley, A. D. O’Connell, H. Wang, J. M. Martinis, and A. M. Cleland, Generation of Fock States in a Superconducting Quantum Circuit, Nature (London) 454, 310 (2008).

[8] L. V. Bishop, J. M. Chow, J. Koch, A. A. Houck, M. H. Devoret, E. Thuneberg, S. M. Girvin, and R. J. Schoelkopf, Nonlinear Response of the Vacuum Rabi Resonance, Nat. Phys. 5, 105 (2009).

[9] D. M. Meekhof, C. Monroe, B. E. King, W. M. Itano, and D. J. Wineland, Generation of Nonclassical Motional States of a Trapped Atom, Phys. Rev. Lett. 76, 1796 (1996).

[10] E. T. Jaynes, in Coherence and Quantum Optics: Proceedings of the 3rd Rochester Conference on Coherence and Quantum Optics, edited by L. Mandel and E. Wolf (Plenum, New York, 1973), p. 35-80.

[11] H. M. Gibbs, Spontaneous Decay of Coherently Excited Rb, Phys. Rev. Lett. 29, 459 (1972).

[12] H. M. Gibbs, Test of Neoclassical Radiation Theory: Incoherent Resonance Fluorescence from a Coherently Excited State, Phys. Rev. A 8, 456 (1973).

[13] A. Imamoğlu, H. Schmidt, G. Woods, and M. Deutsch, Strongly Interacting Photons in a Nonlinear Cavity, Phys. Rev. Lett. 79, 1467 (1997). 
[14] L. Tian and H. J. Carmichael, Quantum Trajectory Simulations of the Two-State Behavior of an Optical Cavity Containing One Atom, Phys. Rev. A 46, R6801 (1992).

[15] K. M. Birnbaum, A. Boca, R. Miller, A. D. Boozer, T. E. Northup, and H. J. Kimble, Photon Blockade in an Optical Cavity with One Trapped Atom, Nature (London) 436, 87 (2005).

[16] C. Lang, D. Bozyigit, C. Eichler, L. Steffen, J. M. Fink, A. A. Abdumalikov, Jr., M. Baur, S. Filipp, M. P. da Silva, A. Blais, and A. Wallraff, Observation of Resonant Photon Blockade at Microwave Frequencies Using Correlation Function Measurements, Phys. Rev. Lett. 106, 243601 (2011).

[17] C. W. Gardiner, Inhibition of Atomic Phase Decays by Squeezed Light: A Direct Effect of Squeezing, Phys. Rev. Lett. 56, 1917 (1986).

[18] A. S. Parkins, P. Zoller, and H. J. Carmichael, Spectral Linewidth Narrowing in a Strongly Coupled Atom-Cavity System via Squeezed-Light Excitation of a "Vacuum" Rabi Resonance, Phys. Rev. A 48, 758 (1993).

[19] K. W. Murch, S. J. Weber, K. M. Beck, E. Ginossar, and I. Siddiqi, Suppression of the Radiative Decay of Atomic Coherence in Squeezed Vacuum, Nature (London) 499, 62 (2013).

[20] A. D. Greentree, C. Tahan, J. H. Cole, and L. C. L. Hollenberg, Quantum Phase Transitions of Light, Nat. Phys. 2, 856 (2006).

[21] M. J. Hartmann, F. G. S. L. Brandão, and M. B. Plenio, Strongly Interacting Polaritons in Coupled Arrays of Cavities, Nat. Phys. 2, 849 (2006).

[22] D. G. Angelakis, M. F. Santos, and S. Bose, PhotonBlockade-Induced Mott Transitions and XY Spin Models in Coupled Cavity Arrays, Phys. Rev. A 76, 031805(R) (2007).

[23] D. Jaksch, C. Bruder, J. I. Cirac, C. W. Gardiner, and P. Zoller, Cold Bosonic Atoms in Optical Lattices, Phys. Rev. Lett. 81, 3108 (1998).

[24] M. Greiner, O. Mandel, T. Esslinger, T. W. Hänsch, and E. Bloch, Quantum Phase Transition from a Superfluid to a Mott Insulator in a Gas of Ultracold Atoms, Nature (London) 415, 39 (2002).

[25] H. J. Carmichael, L. Tian, W. Ren, and P. Alsing, in Cavity Quantum Electrodynamics, edited by P. R. Berman (Academic, Orlando, 1994), p. 381-423.

[26] S. S. Shamilov, A. S. Parkins, M. J. Collett, and H. J. Carmichael, Multi-Photon Blockade and Dressing of the Dressed States, Opt. Commun. 283, 766 (2010).

[27] P. Alsing and H. J. Carmichael, Spontaneous Dressed-State Polarization of a Coupled Atom and Cavity Mode, Quantum Opt. 3, 13 (1991).

[28] P. Alsing, D.-S. Guo, and H. J. Carmichael, Dynamic Stark Effect for the Jaynes-Cummings System, Phys. Rev. A 45, 5135 (1992).

[29] H. J. Carmichael, S. Shamailov, A. S. Parkins, and M. J. Collett, Dissipative Phase Transitions for Photons and a Single Qubit, 18th International Laser Physics Workshop, Barcelona, 2009 (unpublished).

[30] E. Ginossar, L. S. Bishop, D. I. Schuster, and S. M. Girvin, Protocol for High-Fidelity Readout in the Photon Blockade Regime of Circuit QED, Phys. Rev. A 82, 022335 (2010).
[31] V. DeGiorgio and M. O. Scully, Analogy between the Laser Threshold Region and a Second-Order Phase Transition, Phys. Rev. A 2, 1170 (1970).

[32] R. Graham and H. Haken, Laser Light_First Example of a Second-Order Phase Transition Far Away from Thermal Equilibrium, Z. Phys. 237, 31 (1970).

[33] S. Grossmann and P. H. Richter, Laser Threshold and Nonlinear Landau Fluctuation Theory of Phase Transitions, Z. Phys. 242, 458 (1971).

[34] P. R. Rice and H. J. Carmichael, Photon Statistics of a Cavity-QED Laser: A Comment on the Laser-Phase-Transition Analogy, Phys. Rev. A 50, 4318 (1994).

[35] H. M. Gibbs, S. L. McCall, and T. N.C. Venkatessan, Differential Gain and Bistability Using a Sodium-Filled Fabry-Perot Interferometer, Phys. Rev. Lett. 36, 1135 (1976).

[36] R. Bonifacio and L. A. Lugiato, Cooperative Effects and Bistability for Resonance Fluorescence, Opt. Commun. 19, 172 (1976).

[37] R. Bonifacio and L. A. Lugiato, Photon Statistics and Spectrum of Transmitted Light in Optical Bistability, Phys. Rev. Lett. 40, 1023 (1978).

[38] A. T. Rosenberger, L. A. Orozco, H. J. Kimble, and P. D. Drummond, Absorptive Optical Bistability in Two-State Atoms, Phys. Rev. A 43, 6284 (1991).

[39] I. Buluta and F. Nori, Quantum Simulators, Science 326, 108 (2009).

[40] Nature Physics Insight-Quantum Simulation, edited by A. Trabesinger, Nat. Phys. 8, 263 (2012).

[41] A. A. Houck, H. E. Türeci, and J. Koch, On-Chip Quantum Simulation with Superconducting Circuits, Nat. Phys. 8, 292 (2012).

[42] I. Carusotto and C. Ciuti, Quantum Fluids of Light, Rev. Mod. Phys. 85, 299 (2013).

[43] R. Dicke, Coherence in Spontaneous Radiation Processes, Phys. Rev. 93, 99 (1954).

[44] K. Hepp and E. H. Lieb, On the Superradiant Phase Transition for Molecules in a Quantized Radiation Field: The Dicke Maser Model, Ann. Phys. (N.Y.) 76, 360 (1973).

[45] T. K. Wang and F. T. Hioe, Phase Transition in the Dicke Model of Superradiance, Phys. Rev. A 7, 831 (1973).

[46] K. Baumann, C. Guerlin, F. Brennecke, and T. Esslinger, Dicke Quantum Phase Transition with a Superfluid Gas in an Optical Cavity, Nature (London) 464, 1301 (2010).

[47] J. Klaers, J. Schmitt, F. Vewinger, and M. Weitz, BoseEinstein Condensation of Photons in an Optical Microcavity, Nature (London) 468, 545 (2010).

[48] K. Rzążewski, K. Wódkiewicz, and W. Żakowicz, Phase Transitions, Two-Level Atoms, and the $A^{2}$ Term, Phys. Rev. Lett. 35, 432 (1975).

[49] F. Dimer, B. Estienne, A. S. Parkins, and H. J. Carmichael, Proposed Realization of the Dicke-Model Quantum Phase Transition in an Optical Cavity QED System, Phys. Rev. A 75, 013804 (2007).

[50] K. Sheshadri, H. R. Krishnamurthy, R. Pandit, and T. V. Ramakrishnan, Superfluid and Insulating Phases in an Interacting-Boson Model: Mean-Field Theory and the RPA, Europhys. Lett. 22, 257 (1993).

[51] R. Roy and L. Mandel, Dye Laser Statistics and the Phase Transition, Opt. Commun. 23, 306 (1977). 
[52] R. Roy and L. Mandel, Optical Bistability and First Order Phase Transition in a Ring Dye Laser, Opt. Commun. 34, 133 (1980).

[53] L. M. Narducci, D. H. Feng, R. Gilmore, and G. S. Agarwal, Transient and Steady-State Behavior of Collective Atomic Systems Driven by a Classical Field, Phys. Rev. A 18, 1571 (1978).

[54] P. D. Drummond and H. J. Carmichael, Volterra Cycles and the Cooperative Fluorescence Critical Point, Opt. Commun. 27, 160 (1978).

[55] H. J. Carmichael, Analytical and Numerical Results for the Steady State in Cooperative Resonance Fluorescence, J. Phys. B 13, 3551 (1980).

[56] H. Sambe, Steady States and Quasienergies for a QuantumMechanical System in an Oscillating Field, Phys. Rev. A 7, 2203 (1973).

[57] C. R. Stroud, Jr. and E. T. Jaynes, Long-Term Solutions in Semiclassical Radiation Theory, Phys. Rev. A 1, 106 (1970).

[58] S. Ya. Kilin and T. B. Krinitskaya, Single-Atom Phase Bistability in a Fundamental Model of Quantum Optics, J. Opt. Soc. Am. B 8, 2289 (1991).

[59] G.P. Agrawal and H. J. Carmichael, Optical Bistability through Nonlinear Dispersion and Absorption, Phys. Rev. A 19, 2074 (1979).

[60] C. M. Savage and H. J. Carmichael, Single-Atom Optical Bistability, IEEE J. Quantum Electron. 24, 1495 (1988).

[61] Y. Zhu, D. J. Gauthier, S. E. Morin, Q. Wu, H. J. Carmichael, and T. W. Mossberg, Vacuum Rabi Splitting as a Feature of Linear-Dispersion Theory: Analysis and Experimental Observations, Phys. Rev. Lett. 64, 2499 (1990).
[62] J. Gripp, S. L. Mielke, L. A. Orozco, and H. J. Carmichael, Anharmonicity of the Vacuum Rabi Peaks in a Many-Atom System, Phys. Rev. A 54, R3746 (1996).

[63] L. S. Bishop, E. Ginossar, and S. M. Girvin, Response of the Strongly-Driven Jaynes-Cummings Oscillator, Phys. Rev. Lett. 105, 100505 (2010).

[64] R. Bonifacio, M. Gronchi, and L. A. Lugiato, Photon Statistics of a Bistable Absorber, Phys. Rev. A 18, 2266 (1978).

[65] P. D. Drummond and D.F. Walls, Quantum Theory of Optical Bistability. I: Nonlinear Polarisability Model, J. Phys. A 13, 725 (1980).

[66] H. J. Carmichael, An Open Systems Approach to Quantum Optics, Lecture Notes in Physics Monographs, edited by W. Beiglböck (Springer, Berlin, 1993), Vol. 18.

[67] M. A. Armen, A. E. Miller, and H. Mabuchi, Spontaneous Dressed-State Polarization in the Strong Driving Regime of Cavity QED, Phys. Rev. Lett. 103, 173601 (2009).

[68] A. Le Boité, G. Orso, and C. Ciuti, Steady-State Phases and Tunneling-Induced Instabilities in the Driven Dissipative Bose-Hubbard Model, Phys. Rev. Lett. 110, 233601 (2013).

[69] A. Le Boité, G. Orso, and C. Ciuti, Bose-Hubbard Model: Relation between Driven Dissipative Steady States and Equilibrium Quantum Phases, Phys. Rev. A 90, 063821 (2014).

[70] P. D. Drummond and C. W. Gardiner, Generalised P-Representations in Quantum Optics, J. Phys. A 13, 2353 (1980).

[71] H. J. Carmichael, Statistical Methods in Quantum Optics 2: Non-Classical Fields (Springer, Berlin, 2008), Sec. 12.2.

[72] B. Sarabi, A. N. Ramanayaka, A. L. Burin, F. C. Wellstood, and K. D. Osborn, Cavity Quantum Electrodynamics Using a Near-Resonance Two-Level System: Emergence of the Glauber State, Appl. Phys. Lett. 106, 172601 (2015). 